\title{
Age Structure and Growth Pattern of an Endangered Species, Amentotaxus formosana Li
}

\author{
Chinsu Lin ${ }^{1}$, Ming-Hsun Chan ${ }^{2}$, Feng-Sheng Chen $^{1}$ and Ya-Nan Wang ${ }^{2}$ \\ ( ${ }^{1}$ Department of Forestry and Natural Resources, National Chiayi University, Chiayi 60004, Taiwan, China; \\ ${ }^{2}$ Experimental Forest of College of Bio-Resources and Agriculture, National Taiwan University, Nan-Tou Hsien 55743, Taiwan, China)
}

\begin{abstract}
Amentotaxus formosana Li is a native, tolerant, and under-wood species in Taiwan. This particular species is only distributed around the Chachayalaishan and Dawushan areas. Because of its rarity, and the endangered and native properties of the species, we explored the regeneration process of the species in the native environment. This paper, therefore, aims to outline the age distribution and growth characteristics of $A$. formosana in the native stand using the techniques of tree ring analysis. An inventory was conducted in a site at the Chachayalaishan area from 2004 to 2006. According to the inventory, there are 67 trees in the sampling plot among which 16 individual $A$. formosana trees were counted. This is roughly $24 \%$ of the total population. This particular species is distributed around the understory space, while other species such as Litsea spp., Meliosma squamulata Hance, Schefflera octophylla Harms, Elaeocarpus japonicus Sieb et Zucc and Cleyera japonica Thunb are the major species whose canopy occupying the upperstory space of the stand. Results of tree-ring analysis suggest that the age of most of the trees in the sampling plot is between 31 and 90 years old with an average of 56 . For the endangered species, $A$. formosana, age of the individuals varied from 14 and 126 years old and the average was 58 years old. Age distribution of the species was modeled using the Weibull probability density function and was statistically assessed using Sigmaplot statistical software. The estimate of shape parameter is $\mathbf{1 . 4 0 4} 5$ indicating a medium regeneration situation. The diameter growth rate of $A$. formosana is $0.32 \mathrm{~cm}$ and ranks 20 th among the 26 species in the sampling plot. Using the age as a regressor variable, we can predict the diameter increment of $A$. formosana efficiently. The coefficient of determination, $R^{2}$, is $\mathbf{0 . 8 3 2}$ 2. The diameter growth among the trees of the upperstory and understory was competitive. Relationship between the growth change percentage of $A$. formosana and the surrounding hardwoods is negative.
\end{abstract}

Key words: age structure; Amentotaxus formosana; growth pattern; Weibull function.

Lin C, Chan MH, Chen FS, Wang YN (2007). Age structure and growth pattern of an endangered species, Amentotaxus formosana Li. J. Integr. Plant Biol. 49(2), 157-167.

Available online at www.blackwell-synergy.com/links/toc/jipb, www.jipb.net

Received 1 Sept. 2006 Accepted 18 Nov. 2006

Supported by the project "Study on the stand structure and seedling propagation of the Amentotaxus formosana Li" of Taiwan Forest Bureau in 2004 and the project "Spatial information extraction of the stand structure of forest" of the Industrial Technology Research Institute in 2006.

*Author for correspondence.

Tel: +886 49264 2183;

Fax: +886 492659967 ;

E-mail: <mhchan@ntu.edu.tw >.

(C) 2007 Institute of Botany, the Chinese Academy of Sciences doi: 10.1111/j.1672-9072.2007.00429.x
Amentotaxus formosana $\mathrm{Li}$ is a special native species of Taxaceae (sometime included in the Amentotaxaceae) in the south-eastern parts of Taiwan. It is an evergreen shrub or tree and is distributed in native coniferous and broad-leaved mixed forest at altitudes of between 700 and $1500 \mathrm{~m}$. It is limited to a small geographical area (Su 1980). A. formosana likes a moist environment and its growth is strictly constrained by the natural environment, such as physiologically growing under a weak light intensity situation, particularly near the mountain or hill valley or along the stream. Therefore, only small populations of this native species can be found in the field. Because of its rarity, native geographical restriction and historical value (a petrified plant), A. formosana has been classified as an 
endangered species that needs to be protected by law. The habitat of the species has thus been delineated as a natural reserve by the Government.

Yang (1996) indicated that there are many articles focused specifically on the genetic variation, taxonomy and ecology, while few have discussed the population growth and competition of the endangered species in recent years. Wu (1992) and Yang $(1994,1996)$ authored some papers that specifically inventoried the vegetation population of the species in the natural forest using the traditional inventory technique. In these studies, only tree diameter measurements and counts of the individual trees were used for analysis because there was no one in Taiwan who was familiar with non-destructive techniques. Therefore, no $A$. formosana trees have been timber logged for age analysis in the past. Although there have been so many efforts dedicated to genetic culture techniques and plantation building mechanisms for artificial regeneration in the field in recent years, it is necessary to examine the growth trends of such a rare species in view of the age structure. Dendrochronology techniques can now be applied to fit such needs without the use of mechanical logging. Thus, we tried to integrate geospatial technique and dendrochronological technique to map $A$. formosana and other species in a natively mixed forest. It is our intention to understand the regeneration status of $A$. formosana by the way of age distribution modeling and to examine the growth pattern of diameter and height of the species.

\section{Results and Discussion}

\section{Ecological features of the $A$. formosana and hardwoods mixed stand}

\section{Biological diversity and age distribution}

Forest composition in the subtropical mixed forest stand is rich diverse vegetation. This is also evident in the sampling plot of A. formosana and broad-leaved species. Biodiversity and evenness of the stand were assessed according to Shanon-Wiener diversity $\left(H^{\prime}\right)$ and Simpson evenness (1-D) indices. Calculated index value was $H^{\prime}=4.504$ and $1-D=0.379$. There are 67 individual trees in the plot. The trees were classified and belong to 26 species, 26 genuses, and 16 families. We found one was a native conifer, Taiwania cryptomerioides Hayata. Compared to the number of individuals for each species in the stand, $A$. formosana has 16 individuals and is the most dominant, $24 \%$ of the all trees in the plot. The species Meliosma squamulata Hance, Schefflera octophylla Harms, Elaeocarpus japonicus Sieb et Zucc and Cleyera japonica Thunb are subdominant with $27 \%$ of the stand. In this research plot, we found the trees are 56 years old on average and the range is between 14 and 165 years. In particular, all of the individuals of $A$. formosana are between 14 and 126 years, and an average of 58 years. Age distribution of the endangered species is similar to the forest stand. Figure 1 shows the histogram of age distribution for all of the individuals in the site. The individuals were classed every 10-years as a group, after which age distribution could be seen. It looks like a positively skewed Weibull distribution. Briefly, mode age is 40 years and the trees with age class from 31 to 90 years are the major population and there are no young trees in the stand. Table 1 shows the details of the trees in the stand including age, height, diameter increment, wood density and taxonomy information.

\section{Spatial pattern of the height}

The height of each tree is typically the result of a combination of genetic characteristics and environmental impacts. Minimum and maximum height of the stand is 1.3 and $28.0 \mathrm{~m}$, while the individual $A$. formosana's range from 1.3 to $11.8 \mathrm{~m}$. Average height of the stand is 15.3 and $18.0 \mathrm{~m}$ if including and excluding the individuals of $A$. formosana, respectively. Compared to the average height $6.4 \mathrm{~m}$ of the $A$. formosana trees, we can conclude that this endangered species is widely distributed under the main canopy space. If we assign z-axis to represent the height value, $x$ - and $y$-axis to longitudinal and latitudinal coordinates, then the height of each individual could be stereoscopically depicted and the spatial pattern with height trend of the stand could be extracted. In Figure 2, gray dots and black dots stand for the A. formosana and the other species, respectively. A notable trend can be identified, in which most of the $A$. formosana occupy the understory space and rarely reach up to the main story of the stand.

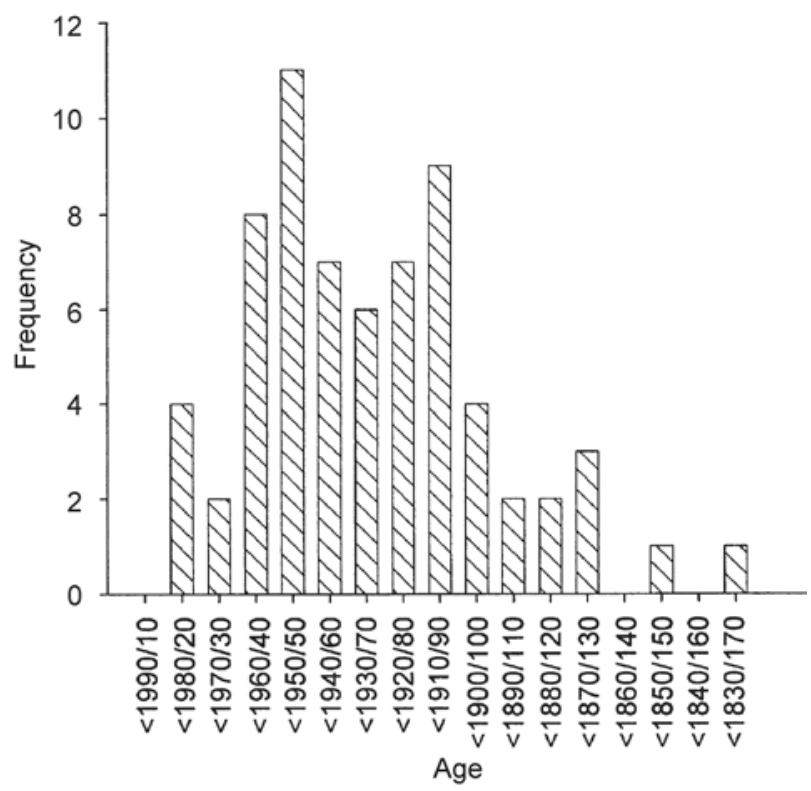

Figure 1. Age distribution of all individuals in the plot $(n=67)$. 


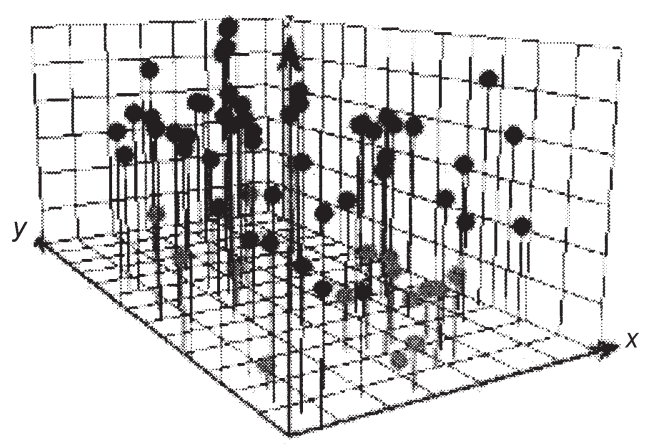

Figure 2. Height structure and profiles of the study site.

\section{Spatial pattern of the diameter}

In this inventory, the diameter is measured at the breast height of the tree. Results showed that the DBH for all of the trees varies dramatically. The largest and smallest diameter is 73.0 $\mathrm{cm}$ and $3.0 \mathrm{~cm}$, and calculated DBH mean is $29.0 \mathrm{~cm}$. $A$. formosana individuals have a relatively small diameter. Their $\mathrm{DBH}$ are between 3 and $28 \mathrm{~cm}$ and have a mean of $11.7 \mathrm{~cm}$.

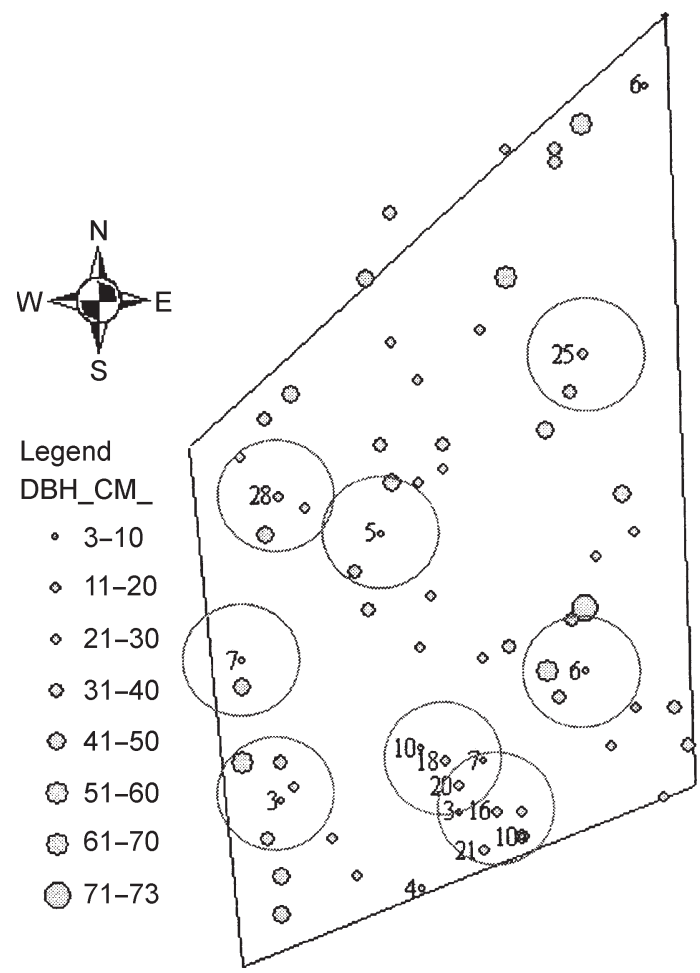

Figure 3. Spatial distribution of the grouped diameter classes of the study site. Diameters of the trees in the plot range from 3 to $73 \mathrm{~cm}$.

Number next to the symbol shows the actual diameter of each Amentotaxus formosana. Each circle delineates the boundary with 5-meter radius from the $A$. formosana. The circles define the possible candidates for growth competition.
Figure 3 uses graduate symbols with varying sizes of circles representing the $\mathrm{DBH}$ of each tree. It is clear that the circle position demonstrates the geospatial location of trees. Particularly, a specific number denoting the real diameter of $A$. formosana is next to the location of the individual. Therefore, the spatial pattern of diameter is distinct. In the sampling plot, most of the endangered species are close to each other and near the middle bottom of the site. These individuals seem to grow by space competition with each other because the trees with larger diameter are taller than the smaller ones. Although some of the A. formosana distribute separately, we still found that these individuals are distributed close to other hardwood species. Hardwood species usually have a wide crown canopy. For the individuals of those hardwood species that take over the upperstory space of the stand, they might depress the surrounding individuals growing understory. Unfortunately, $A$. formosana is the depressed. We therefore delineate some groups showing such spatial relations between $A$. formosana and hardwood species. The possible growth competition for the individuals between the upper- and lower-story will be discussed later. DBH data of all trees were summarized and grouped into a histogram in Figure 4. The mode diameter is the $40-\mathrm{cm}$ class.

\section{Insides the age structure and growth pattern of $A$.} formosana

\section{Age distribution and the regeneration potential}

Although $A$. formosana is dominant in the sampling area, the population has 16 species, which is not enough for modeling the age distribution using the maximum likelihood method. We therefore tried to collect more individuals of $A$. formosana near the sampling area. We finally got an extra 18 individuals (the dots locate on northeastern position of the sampling plot in the figure showing topographic features of the study site) and

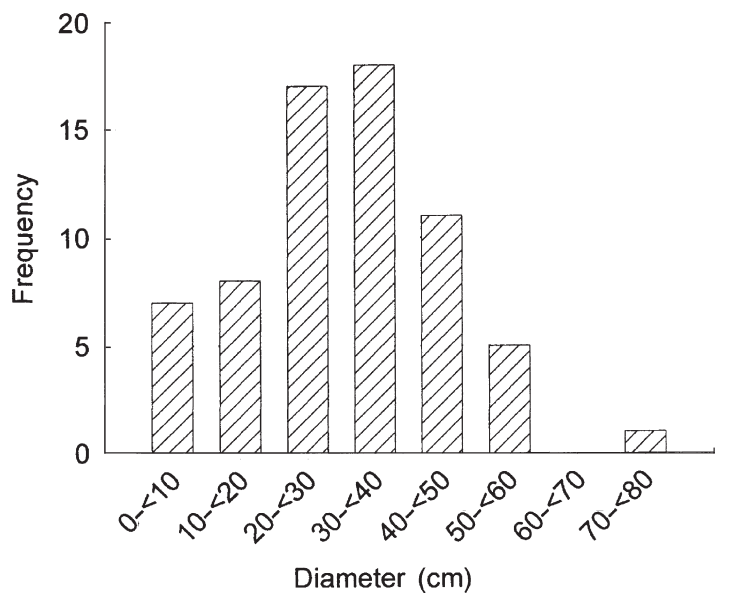

Figure 4. Diameter distribution of all individuals in the plot $(n=67)$. 
Table 1. Species and general growth characteristics of the sample plot

\begin{tabular}{|c|c|c|c|c|c|c|c|c|c|}
\hline Species & Family & $N$ & $\begin{array}{l}\text { Wood density } \\
\qquad\left(\mathrm{g} / \mathrm{cm}^{3}\right)^{\mathrm{a}}\end{array}$ & $\begin{array}{c}\text { Diameter } \\
\text { increment } \\
(\mathrm{cm} / \mathrm{yr})^{\mathrm{a}}\end{array}$ & $\begin{array}{c}\text { Mean } \\
\text { height } \\
(\mathrm{m})\end{array}$ & $\begin{array}{l}\text { Max. } \\
\text { height } \\
(\mathrm{m})\end{array}$ & $\begin{array}{c}\text { Min. } \\
\text { age } \\
(\mathrm{yr}) \\
\end{array}$ & $\begin{array}{c}\text { Max. } \\
\text { age } \\
(\mathrm{yr}) \\
\end{array}$ & $\begin{array}{c}\text { Mean } \\
\text { age } \\
(\mathrm{yr}) \\
\end{array}$ \\
\hline \multicolumn{10}{|l|}{ Emergent } \\
\hline Microtropis fokienensis & Celastraceae & 1 & 0.72 & 0.25 & 24.0 & 24.0 & 107 & 107 & 107 \\
\hline Myrsine seguinii & Myrsinaceae & 1 & 0.84 & 0.48 & 28.0 & 28.0 & 91 & 91 & 91 \\
\hline Sloanea formosana & Elaeocarpaceae & 1 & 0.67 & 0.51 & 26.6 & 26.6 & 27 & 27 & 27 \\
\hline \multicolumn{10}{|l|}{ Main canopy } \\
\hline Ardisia sieboldii & Myrsinaceae & 1 & 0.68 & 0.39 & 15.0 & 15.0 & 37 & 37 & 37 \\
\hline Castanopsis formosana & Fagaceae & 1 & 1.05 & 0.39 & 20.0 & 20.0 & 42 & 42 & 42 \\
\hline Cinnamomun micranthum & Lauraceae & 1 & 0.87 & 0.32 & 19.2 & 19.2 & 58 & 58 & 58 \\
\hline Cleyera japonica & Theaceae & 4 & $0.83 \pm 0.09$ & $0.3 \pm 0.09$ & 18.1 & 21.0 & 81 & 127 & 94 \\
\hline Cyclobalanopsis hypophaea & Fagaceae & 1 & 1.01 & 0.26 & 20.0 & 20.0 & 15 & 15 & 15 \\
\hline Cyryptocarya chinensis & Lauraceae & 3 & $0.70 \pm 0.12$ & $0.43 \pm 0.12$ & 16.0 & 19.0 & 38 & 54 & 44 \\
\hline Daphniphyllum glaucescens & Daphniphyllaceae & 1 & 0.73 & 0.41 & 17.0 & 17.0 & 56 & 56 & 56 \\
\hline Elaeocarpus japonicus & Elaeocaroaceae & 5 & $0.84 \pm 0.12$ & $0.61 \pm 0.20$ & 18.0 & 20.0 & 29 & 90 & 59 \\
\hline Engelhardtia formosana & Juglandaceae & 1 & 0.53 & 0.36 & 16.0 & 16.0 & 55 & 55 & 55 \\
\hline Garcinia multiflora & Guttiferae & 1 & 0.83 & 0.28 & 18.3 & 18.3 & 62 & 62 & 62 \\
\hline Gordonia axillaris & Theaceae & 2 & $0.96 \pm 0.00$ & $0.31 \pm 0.04$ & 17.9 & 20.0 & 67 & 122 & 95 \\
\hline Ilex formosae & Aquifoliaceae & 2 & $0.72 \pm 0.40$ & $0.48 \pm 0.44$ & 19.5 & 24.0 & 34 & 75 & 55 \\
\hline Litsea akoensis & Lauraceae & 2 & $0.87 \pm 0.01$ & $0.34 \pm 0.01$ & 14.5 & 16.0 & 15 & 57 & 36 \\
\hline Litsea acuminata & Lauraceae & 2 & $0.70 \pm 0.02$ & $0.58 \pm 0.20$ & 15.0 & 20.0 & 39 & 68 & 54 \\
\hline Meliosma squamulata & Sabiaceae & 7 & $0.69 \pm 0.10$ & $0.28 \pm 0.05$ & 17.8 & 21.0 & 32 & 165 & 98 \\
\hline Neolitsea acuminatissima & Lauraceae & 1 & 0.86 & 0.42 & 19.0 & 19.0 & 71 & 71 & 71 \\
\hline Syzygium formosanum & Myrtaceae & 1 & 0.94 & 0.16 & 23.1 & 23.1 & 46 & 46 & 46 \\
\hline Schima superba & Theaceae & 3 & $0.85 \pm 0.06$ & $0.48 \pm 0.30$ & 22.7 & 25.0 & 34 & 90 & 68 \\
\hline Schefflera octophylla & Araliaceae & 6 & $0.67 \pm 0.12$ & $0.68 \pm 0.10$ & 18.1 & 21.0 & 42 & 70 & 54 \\
\hline Taiwania cryptomerioides & Taxodiaceae & 1 & 0.97 & 0.18 & 12.2 & 12.2 & 31 & 31 & 31 \\
\hline Turpinia ternata & Staphyleaceae & 1 & 0.81 & 0.38 & 14.0 & 14.0 & 28 & 28 & 28 \\
\hline Wendlandia formosana & Rubiaceae & 1 & 0.77 & 0.61 & 11.2 & 11.2 & 18 & 18 & 18 \\
\hline \multicolumn{10}{|l|}{ Understory } \\
\hline Amentotaxus formosana & Amentotaxaceae & 16 & $0.74 \pm 0.20$ & $0.32 \pm 0.11$ & 6.4 & 11.0 & 14 & 126 & 58 \\
\hline
\end{tabular}

avalues followed the symbol " \pm " indicate the standard deviation (SD) of the data; the other species have no SD due to no replications in the plot.

included them with the original individuals in the dataset. All the trees $(n=34)$ were also grouped with a 10 -year span and accumulated to form the age histogram of $A$. formosana. The dataset was then used for estimating the parameters of variant distribution models, such as Gaussian, Log Normal, and Weibull functions. The fitted distribution curves of those models were integrated into Figure 5. Parameter estimates of the models were statistically tested. The shape parameter estimates of the Gaussian empirical model and Log Normal empirical model are $b=37.0050$ and $b=0.7676$, respectively; these two estimates are tested not significantly at the probability level of 0.05 (Tables 2, 3). We therefore conclude that the age distribution of $A$. formosana could not be modeled well using Gaussian or Log Normal function. Fortunately, the scale $(\alpha)$, shape $(\gamma)$,

Table 2. Significance test of the coefficients for the fitted Gaussian model ${ }^{\dagger}$

\begin{tabular}{lrrrr}
\hline Model parameters & Estimated coefficient & SE of coefficient & \multicolumn{1}{c}{$t$-value } & \multicolumn{1}{c}{$P$-value } \\
\hline$a$ & 4.8412 & 2.3471 & 2.0626 & 0.0661 \\
$b(\sigma)$ & 37.0050 & 20.9399 & 1.7672 & 0.1076 \\
$x_{0}(\mu)$ & 58.7971 & 7.6062 & 7.7302 & $10.1192<$ \\
$y_{0}$ & -0.5156 & 2.5160 & -0.2049 & 1.0906 \\
\hline
\end{tabular}

${ }^{\dagger}$ Empirical model is in the form of equation (1). P-value indicates that the $t$-value is significant at the specific probability. 
Table 3. Significance test of the coefficients for the fitted Log Normal model ${ }^{\dagger}$

\begin{tabular}{lrrrrr}
\hline Model parameters & Estimated coefficient & SE of coefficient & \multicolumn{1}{c}{$t$-value } & \multicolumn{1}{c}{$P$-value } \\
\hline$a$ & 6.0350 & 3.6911 & 1.6350 & 0.1331 & $40.7822<$ \\
$b(\sigma)$ & 0.7676 & 0.4931 & 1.5569 & 0.1506 & $27.8926<$ \\
$x_{0}(\mu)$ & 42.8890 & 5.8999 & 7.2694 & $<0.0001$ & 1.7866 \\
$y_{0}$ & -1.3650 & 3.9579 & -0.3449 & 0.7373 \\
\hline
\end{tabular}

${ }^{\dagger}$ Empirical model is in the form of equation (2).

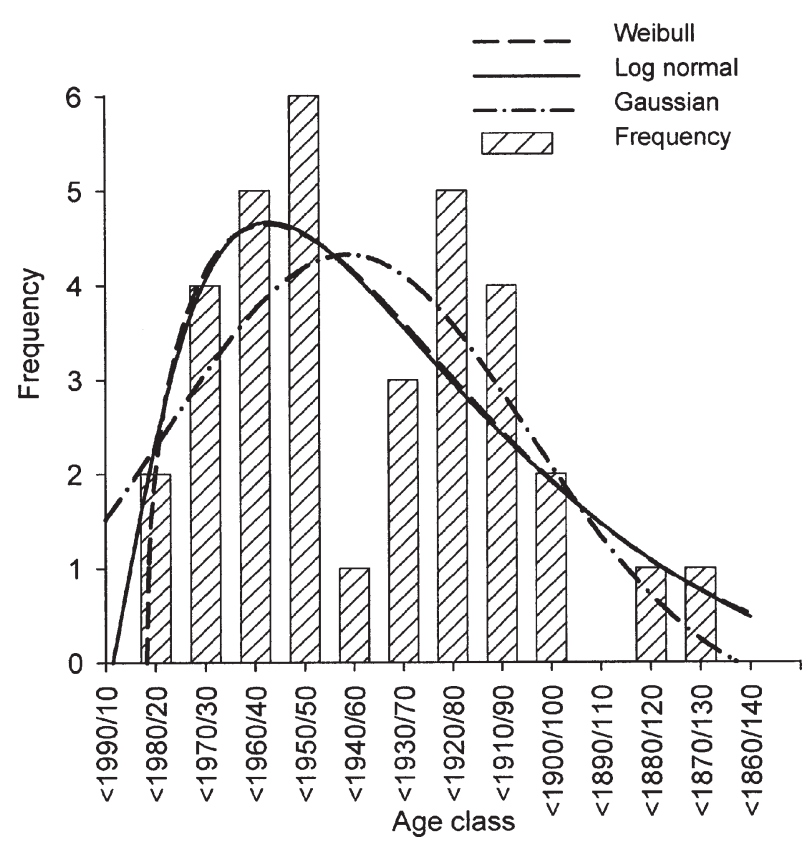

Figure 5. Model fitted age distribution of Amentotaxus formosana $(n=34)$.

and position $(\mu)$ parameters of the Weibull are all well estimated and the estimates are $b=4.9538, c=1.4045$, and $x_{0}=42.5903$, respectively. Those coefficients are statistically significant at the probability level of 0.05 as demonstrated in Table 4 . Results indicate that the Weibull function is suitable for modeling the age distribution of $A$. formosana. Specifically, the estimate of shape parameter $(c=1.4045)$ falls into the region of $1<\gamma<2.6$. Thus, we can conclude the individual $A$. formosana has a positively skewed Weibull distribution and the regeneration status of the endangered species is medium.

$$
\begin{aligned}
& f=y_{0}+a \times \exp \left(-0.5 \times \frac{x-x_{0}}{b}\right)^{2} \\
& \left.f=y_{0}+a \times \exp \left(-0.5 \times\left(\frac{\ln \left(x / x_{0}\right)}{b}\right)\right)^{2}\right)
\end{aligned}
$$

$$
\begin{aligned}
& f=y_{0}+a \times\left(\frac{c-1}{c}\right)^{\frac{1-c}{c}} \times\left|\frac{x-x_{0}}{b}+\left(\frac{c-1}{c}\right)^{\frac{1}{c}}\right|^{(c-1)} \\
& \times \exp \left(-\left|\frac{x-x_{0}}{b}+\left(\frac{c-1}{c}\right)^{\frac{1}{c}}\right|^{c}\right)+\frac{c-1}{c}
\end{aligned}
$$

\section{Growth pattern and competition}

\section{Modeling of diameter and height growth and assessment}

The diameter growth of each individual $A$. formosana is depicted in Figure 6A. Although the DBH increment is slightly different from other individuals, those variant $\mathrm{DBH}$ values $(y)$ are then averaged according to the ages $(x)$ to derive a generalized linear model. Using the diameter growth model, all of the individual $\mathrm{DBH}$ were predicted based on age and then plotted versus the observed value for assessing the model suitability. The results in Figure 6B show that the diameter growth of $A$. formosana is highly correlated with the variable age. The coefficient of determination $\left(R^{2}\right)$ of the fitted model is 0.7549 , showing good efficiency of the generalized diameter growth model of $A$. formosana. We found that it is difficult to correlate the height with the diameter and with the age of $A$. formosana. Figure 6C, D showed small $R^{2}$ could be accomplished for height estimation using diameter or age.

\section{Competition}

Based on the geospatial neighborhood concept, we chose some individuals of the hardwood species that grew around the $A$. formosana for competition analysis. Neighbors were picked if they were in the scope of a 5-meter radius distance of the $A$. formosana. Circles in Figure 3 depict the possible candidates. The numbers of trees are respectively $n=13$ and $n=15$ for $A$. formosana and the hardwood species. A 5-year scaled growth change percentage was applied to analyze the growth pattern of those individuals for both categories. In Figure 7, the real and dash curve indicates GC\% trend of $A$. formosana and the hardwood category and the number sequence lines in the upper and bottom of the figure denote the number of individuals that trees germinated in the corresponding years. Hence, there were 3 individuals of hardwood, only one tree of $A$. formosana 

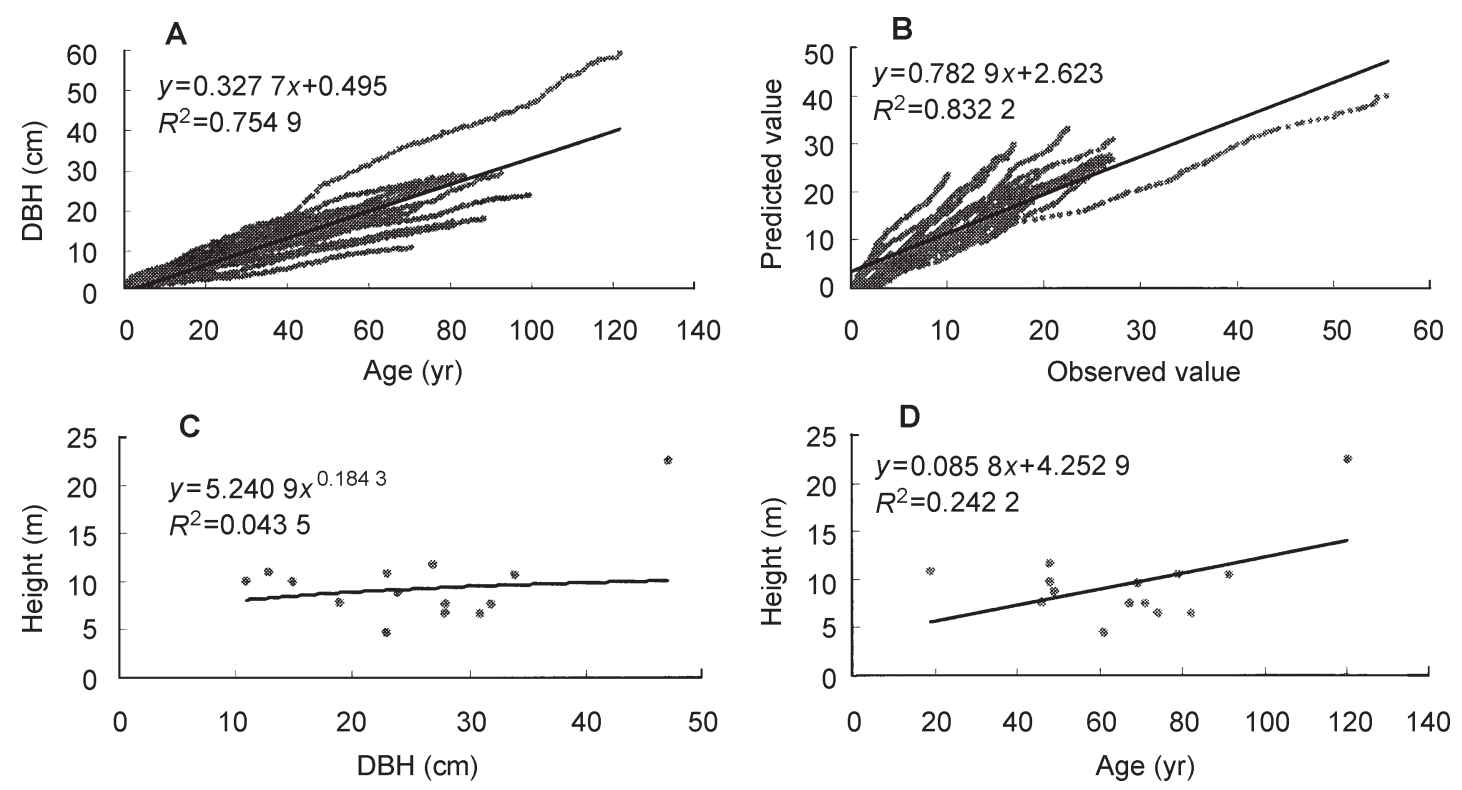

Figure 6. Modeling of diameter increment and height growth for Amentotaxus formosana.

(A) Diameter growth model.

(B) Assessment of diameter growth estimation.

(C) Relation of height vs diameter.

(D) Relation of height vs age.

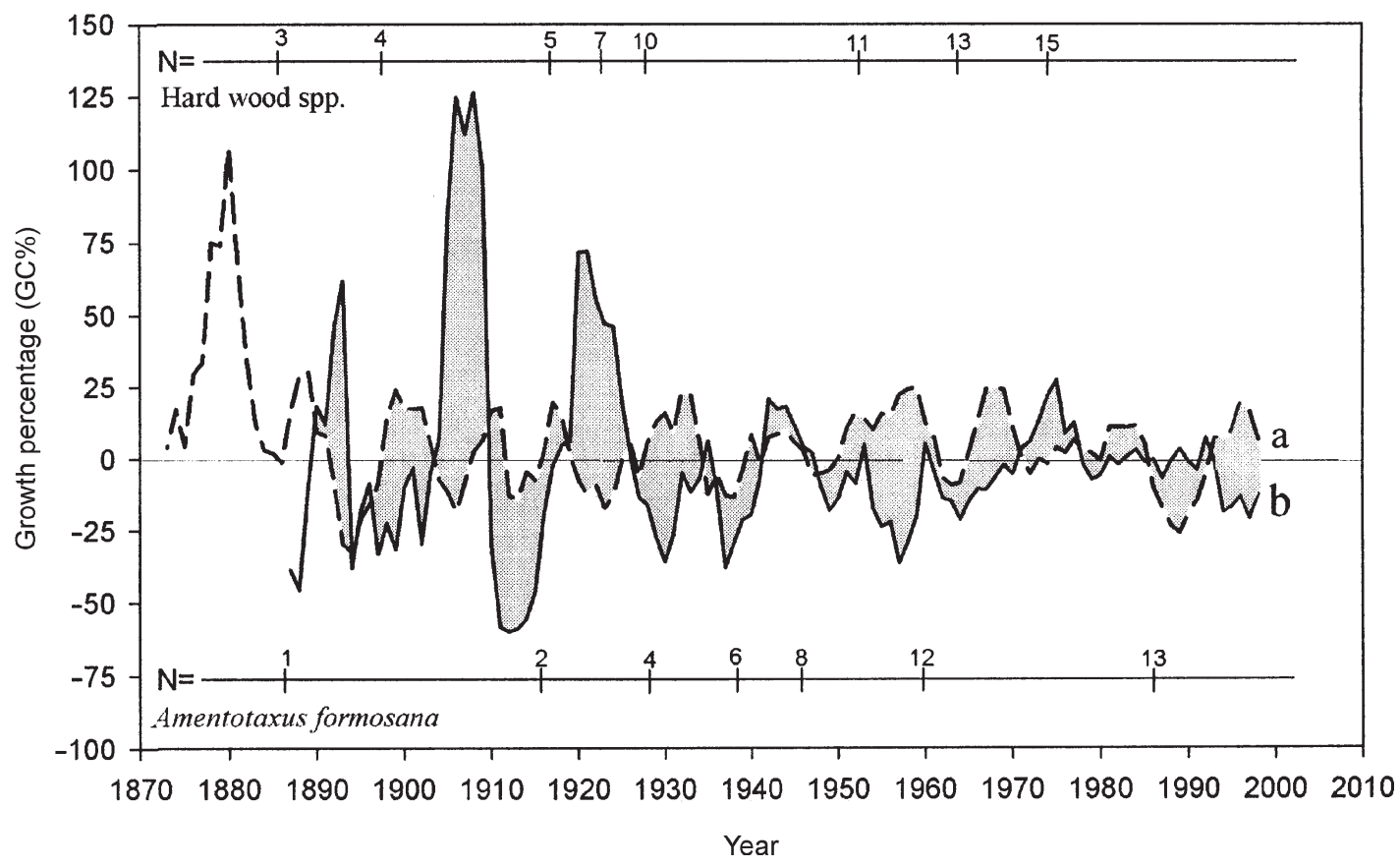

Figure 7. Growth competition between the Amentotaxus formosana and neighboring hardwood trees.

The real and dash curve indicates GC\% trend of $A$. formosana and the hardwood category and the number sequence lines in the upper and bottom of the figure denote the number of individuals. 
Table 4. Significance test of the coefficients for the fitted Weibull model ${ }^{\dagger}$

\begin{tabular}{|c|c|c|c|c|c|}
\hline Model parameters & Estimated coefficient & SE of coefficient & $t$-value & $P$-value & VIF \\
\hline$a$ & 59.3297 & 21.1226 & 2.8088 & 0.0204 & $9.2181<$ \\
\hline$b(\sigma)$ & 4.9538 & 1.6024 & 3.0915 & 0.0129 & $6.0945<$ \\
\hline$c(\gamma)$ & 1.4045 & 0.4818 & 2.9152 & 0.0172 & $26.3627<$ \\
\hline$x_{0}(\mu)$ & 42.5903 & 13.5122 & 3.1520 & 0.0117 & $20.5432<$ \\
\hline$y_{0}$ & -0.3053 & 1.4691 & -0.2078 & 0.8400 & $12.6934<$ \\
\hline
\end{tabular}

${ }^{\dagger}$ Empirical model is in the form of equation (3).

growth of both categorical species change dramatically and the changes are obviously correlated. Correlation coefficients between the GC\% of $A$. formosana and the hardwood are $r=-$ $0.34(P<0.05)$ and $r=-0.27(P<0.04)$ for the data from the period of 1950-2004 and 1887-2004. This indicates that the GC\% between $A$. formosana and the hardwood is statistically significant and their relationship is negative.

\section{Conclusion}

Results of this study demonstrate that the endangered species, A. formosana, is a tolerant species growing in the understory of mixed forest in Taiwan. Diameter growth of the species is strongly dependent on the age and is accurately predictable while the height growth is not predictable using age or diameter of the tree, which is because of the influence of complicated environmental factors. The observed rate of diameter increment is $0.32 \mathrm{~cm}$ per year and the standard deviation is 0 . $11 \mathrm{~cm}$. The diameter growth rate is less than most of the hardwood species in the forest stand. The individuals of the species are between 14 and 126 years and their chronology data were statistically tested. It is concluded that age distribution is positively right skewed indicating that the species is under medium regeneration status. Finally, the relationship between the growth change percentage of $A$. formosana and the hardwood species is negatively significant. This seems to indicate that the growth rate of $A$. formosana will go slowly when the surrounding upperstory hardwoods grow very well, and vice versa. In the native forest, there is rarely impact from human activities or animals. Therefore, the most important factor to disturb the growth of trees might be the intensive weather as typhoons invade Taiwan very frequently. Typhoons usually destroy the emergent or main canopy of the forest and lots of branches and leaves are thus removed. This leads to a reduction in tree growth in the coming years until they recover from damage.

\section{Materials and Methods}

\section{Sampling and Survey}

Amentotaxus formosana Li. is native and found to be only

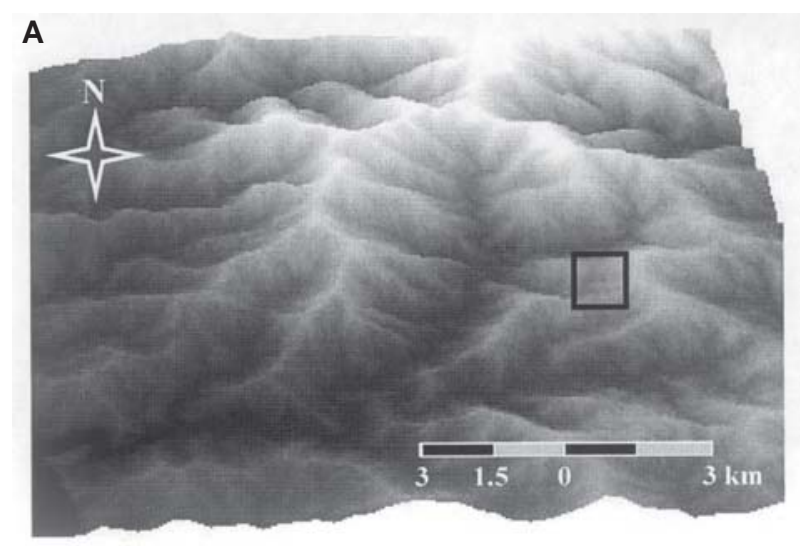

B

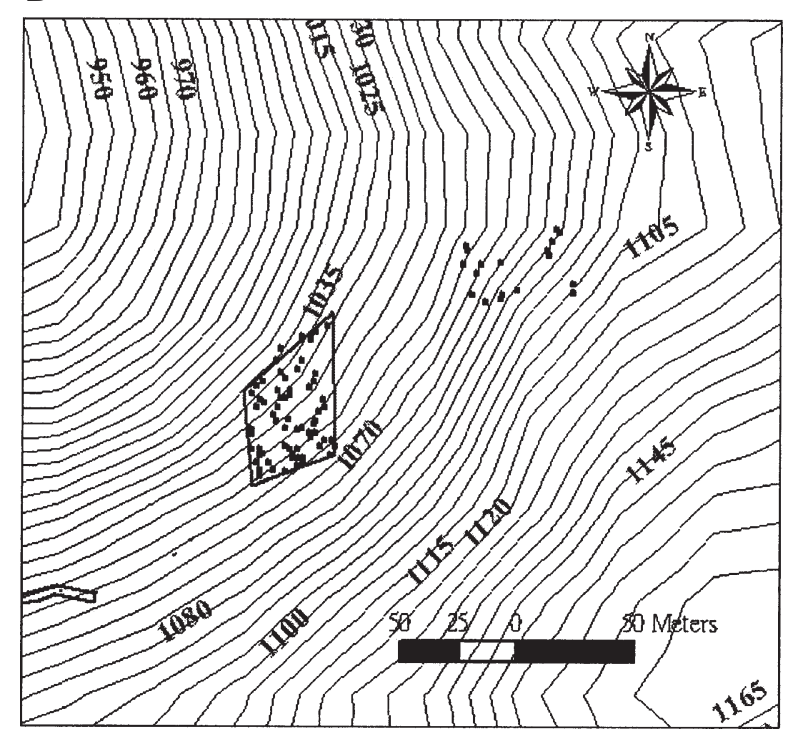

Figure 8. Topographic features of the study site.

(A) Square box in plot, indicating the specific sampling area for research whose upper-left and lower-right geographical coordinate (longitude, latitude) is $\left(22^{\circ} 20^{\prime} 32.7171^{\prime \prime} \mathrm{E}, 120^{\circ} 45^{\prime} 8.7613^{\prime \prime} \mathrm{N}\right)$ and (2220'14.877 1" E, $120^{\circ} 45^{\prime} 36.7521$ 1" N), respectively.

(B) Contours in plot, which are delineated 5-meter altitudinal scale of the area where the elevation ranges from 950 to $1165 \mathrm{~m}$ and with a northwestern aspect.

Polygon with dots in the center position of plot is the sampling plot whose area is $0.22 \mathrm{hm}^{2}$, and the dots denote the location of the trees. 
distributed around the Chachayalaishan near the watershed of Dawu river and Fangshan river of southern Taiwan. Historical records show that the total distribution area of $A$. formosana is approximately $5000 \mathrm{~km}^{2}$, while the actual area occupied by the species is estimated to be not greater than $500 \mathrm{~km}^{2}$ (Yang 1996). Figure 8 shows the particular site chosen as the research plot for examining the stand structure of tree age and growth competition between the $A$. formosana and some other species. The geographical location of the sampling area and each tree in the plot were surveyed by a Trimble GPS receiver and an electronic theodolite. Digital coordinates of those trees were then imported into a geographic information system software, ArcGIS, for event location mapping and for showing all of the individuals and for further spatial analysis (Figure 9). After the crew survey, we found that the $A$. formosana was distributed around the moist valley. Although foresters in Taiwan agreed that there are not many seedlings in the habitat of A. formosana, we found that some seedlings grow next to rocks and that old $A$. formosana trees were decaying and sprouting from the trunk base (Figure 10).

\section{Dendrochronology technique}

\section{Increment cores preparation and soft X-ray image analysis}

We took one core from each individual of $A$. formosana and the neighboring trees in the sample plot. Tree-ring samples were first immersed with $98 \%$ methanol solution, and then distilled water was replaced until the solution was clean. Preprocessed core samples were then embedded into a wood material with glair gel and cut into 2-mm thick and 5-mm wide samples with a highly accurate plane-table saw. The moisture of tree-ring samples was adjusted to $12-15 \%$ in a thermostat that also had stable humidity. An X-ray image was then taken for image processing and analysis. The soft $X$-ray machine (HP CABINE Xray system series Model 43855B) used in this study was made by Hewlett Packard. Two requests were made for the shooting: (1) X-ray shooting distance set to $62.5 \mathrm{~cm}$ from the samples; the range of the negative film was $35 \mathrm{~cm} \times 43 \mathrm{~cm}$ (13 in $\times 16$ in) (KODAK Industrex M100 film, 50 ready pack II); (2) the power of X-ray was $9 \mathrm{PkeV}, 3 \mathrm{~mA}$, for $7 \mathrm{~min}$. Finally, the $\mathrm{X}$-ray image

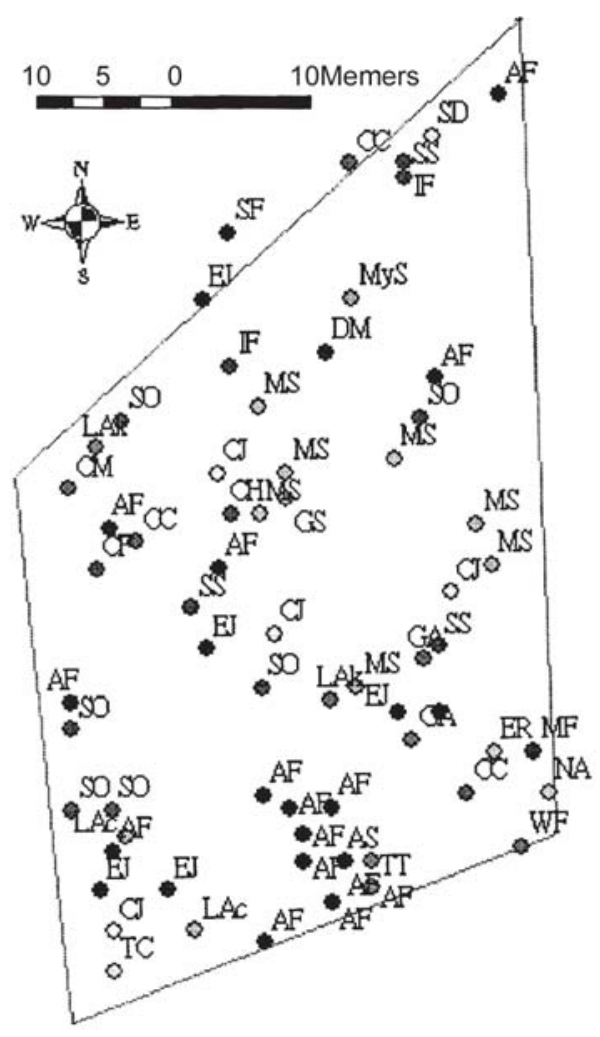

\begin{tabular}{ll}
\hline Abbreviation & \multicolumn{1}{c}{ Species } \\
\hline AF & Amentotaxus fomosana \\
MS & Meliosma squamulata \\
SO & Schefflera octphylla \\
EJ & Elaeocarpus japonicus \\
CJ & Cleyera japonica \\
SS & Schima superba \\
GA & Gordonia axillaris \\
IF & Ilex fmosana \\
DM & Daphniphyllum membranaceum \\
SD & Sloanea dasycarpa \\
CF & Castanopsis formosana \\
CH & Cyclobalanopsis hypohaea \\
MF & Microtropis fokienensis \\
GS & Garcinia subelliptica \\
ER & Engelhardtia roxburghiana \\
LAc & Litsea acumina \\
LAk & Litsea akoensis \\
CC & Cryptocarya chinensis \\
CM & Cinnamomum micranthum \\
NA & Neolitsea acuminatissima \\
AS & Ardisia sieboldii \\
MyS & Myrsine sequinii \\
SF & Taiwaniam formosanum \\
WF & \\
TT & \\
TC & Cryptomerioides \\
\hline & \\
\hline
\end{tabular}

Figure 9. Distribution of the trees in the sample area.

Each point represents the position of trees in the stand and labeled with the abbreviation of the species name. 
A

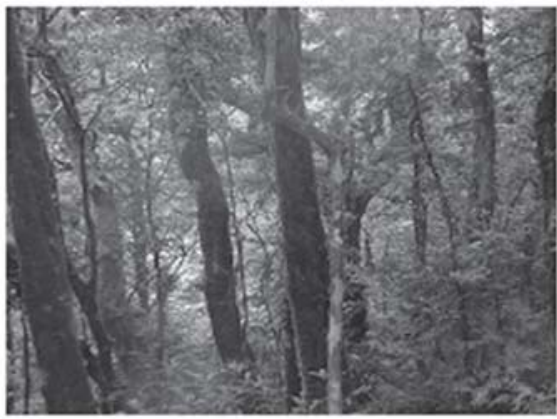

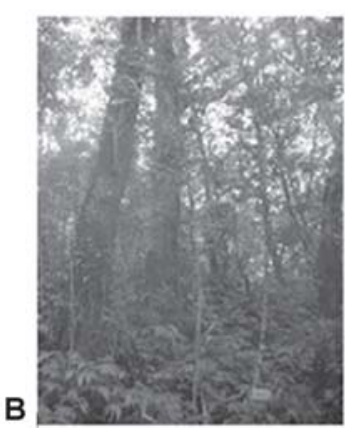

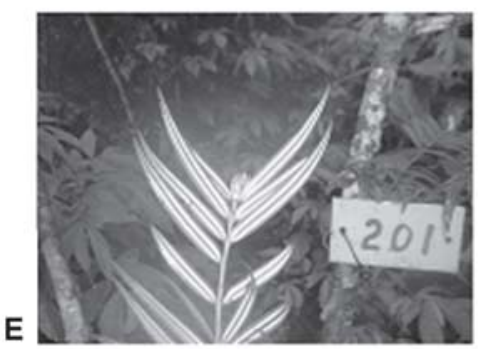

Figure 10. In situ illustrations of the study site.

(A) and (B) show a view of the sample plot.

(C) Some sprouts start to grow from the base of the tree.

(D) Some new individuals grew from seed germination.

(E) A close-up picture of Amentotaxus formosana.

was used to analyze tree-ring width and density of the cores samples by using five different softwares: Image Pro Plus 4.5. 0.29 version for Windows, Sigma Plot 2001 for Windows Version 7.0, TsapWin Professional 0.23b Version 5.1, and Tree Ring X-ray 32 (Nobori 1989). Procedure for integrating those kinds of softwares was developed by Chan et al. (2004).

\section{Tree-ring cross-dating and series standardization}

Growth-ring dating was executed by incorporating the visioning method and statistic program analysis. Visual cross-dating was done by the skeleton-plot process (Stokes and Smiley 1968) and Image-Pro Plus. After that, cross-dating of the treering series was achieved using dendrochronology program COFECHA (Holmes et al. 1986). In this study, the standardization of ring-width series was carried out using the program ARSTAN. This involves fitting the observed ring-width series to a de-trend curve and computing the tree-ring width index (It), which divides the observed ring widths (Rt) by the expected value (Gt). The value Gt was estimated by using cubic spline smooth function (CSSF) and bi-weight robust mean method (BRMM) in the program. CSSF removes the trends resulting from different ages of trees and creates annals of exponent series (Cook and Peter 1981). BRMM removes the effects of external disturbance factors in the stand and uses the mean annals.

\section{Averaging percentage growth change}

Natural disturbances in forests might cause serious vegetation damages and the release of growing space. They are also a source of mortality for some individuals and a source of establishment and growth for others, and the ongoing process of death and replacement has a profound effect on forest structure and composition (Oliver and Larson 1996; Rentch et al. 2002). Percentage of growth change (\%GC) (equation 4) where $M_{1}$ and $M_{2}$ represent the averaged radial growth for the first period of $5 \mathrm{yr}$ and the second period of the following 5 years was proven to be useful in forest dynamic researches and was applied to examine the effects of climate and typhoons on the old-growth species in North Taiwan. Although Rentch et al. (2002) showed that many researches suggested 1-20 yr variant growth lag or years interval for \%GC determination. A 5year ring-width mean suggested by Lorimer and Frelich (1989) was applied to determine canopy disturbance rates in this paper.

$$
\% G C=\frac{M_{2}-M_{1}}{M_{1}} \times 100
$$

\section{Weibull probability function for stand age structure analysis}

For empirical modeling research, we usually apply a particular 
distribution function to fit surveyed data. In practice, only one specific model could be examined for each trial of test. For example, if random variable $X$ is Gaussian distribution (equation 5), denoted $N\left(\mu, \sigma^{2}\right)$, the observed data have to satisfy the maximum likelihood estimates $\hat{\mu}=\bar{X}_{n}$ and $\hat{\sigma}^{2}=\frac{1}{n} \sum_{i=1}^{n}\left(X_{i}-\bar{X}_{n}\right)$; and if the observed data are maximum likelihood estimated with $\hat{\lambda}=\frac{1}{\bar{X}_{n}}$ then they have exponential distribution (equation 6).

$$
\begin{gathered}
f(x)=\frac{1}{\sqrt{2 \pi} \sigma} e^{-(x-\mu)^{2} /\left(2 \sigma^{2}\right)} \text { for } \mu \in R, \sigma>0, \text { and } x \in R \\
f(x)=\lambda e^{-\lambda x} \text { for } \lambda>0 \text { and } x>0
\end{gathered}
$$

Distributions of ecological phenomena are usually dependent on the environmental and the interior factors of organism. For instance, regeneration status of vegetation might change along a time series and it is possible to reveal exponential, normal, or skewed distribution. Regular distributions like the pre-discussed ones are so conditionally restricted and are, therefore, not suitable for such needs. Fortunately, Weibull function (equation 7) is a versatile distribution and is one of the most widely used lifetime distributions in reliability engineering. It can take on the characteristics of other types of distributions based on the value of the shape parameter, $\gamma$. Hence, it is very convenient for ecological applications where the data distribution is unknown and needs to be empirically explored. Some researches about the analysis of forest diameter structure conducted by Bailey and Dell (1973), Tanouchi and Yamamoto (1995), and Yang (1994) have proven the powerful flexibility and suitability of Weibull function for ecological applications. Theoretically, the three-parameter Weibull probability density function $(p d f)$ is given by:

$$
\begin{aligned}
& f(x)=\frac{\gamma}{\alpha}\left(\frac{x-\mu}{\alpha}\right)^{\gamma-1} \exp \left(-\left(\frac{x-\mu}{\alpha}\right)^{\gamma}\right) \\
& \text { for } x \geqslant 0 \text { or } \mu, \gamma>0, \text { and } \alpha>0
\end{aligned}
$$

Where $x$ is the variable observation, such as dbh or age, $\gamma$ is the shape parameter, $\alpha$ is the scale parameter and $\mu$ is the location parameter.

In this study, the parameters of Weibull pdf distribution were estimated with the statistical package Sigmaplot using the maximum likelihood method. However, only one specific value could be estimated for each of the parameters based on the findings of Weibull function in ReliaSoft (2006) and Bailey and Dell (1973). It is concluded that the respective estimates of $\gamma$ have the following meanings:

a) For $0<\gamma<1$, it gives an exponential distribution and indicates there are abundant young individuals in the stand plot and regeneration status of the species is very good; b) For $1<\gamma<2.6$, a positively skewed Weibull pdf distribution (has a right tail) is suggested. It indicates a right-skewed distribution of the age and means a few young individuals compared to the individuals of average age, and also means that the regeneration status is medium;

c) If $2.6<\gamma<3.7$, the coefficient of skewness approaches zero and stands for the Weibull pdf distribution may approximate the normal pdf (has no tail); and

d) For $\gamma<3.7$, it is negatively skewed distribution (has left tail) and indicates a poor regeneration status.

\section{References}

Bailey RL, Dell TR (1973). Quantifying diameter distributions with the Weibull function. For. Sci. 19, 97-104.

Cook ER, Peter WK (1981). The smooth spline: a new approach to standardizing forest interior tree-ring width series for dendroclimatic studies. Tree-Ring Bull. 41, 45-53.

Chan MH, Wang YN, Wang SY (2004). Application of soft X-ray image analysis to the width and density of tree-rings of Zelkova serrata, Cinnamomum camphora and Michelia compressa natural forest. Quart. J. Chin. For. 37, 379-392 (in Chinese with an English abstract).

Holmes RL, Adams RK, Fritts HC (1986). Tree-Ring Chronologies of Western North America: California, Eastern Oregon and Northern Great Basin with Procedures Used in the Chronology Development Work Including Users Manuals for Computer Programs COFECHA and ARSTAN. Laboratory of Tree-Ring Research, University of Arizona, Tucson.

Lorimer CG, Frelich LE (1989). A methodology for estimating canopy disturbance frequency and intensity in dense temperate forests. Can. J. For. Res. 19, 651-663.

Nobori Y (1989). Shin-Nenrin Kaiseki Program Tree Ring Analysis Program. ver. 2.1. Oij Co. Institute of Forestry Improvement. Kuriyama, Hokkaido.

Oliver CD, Larson BC (1996). Forest Stand Dynamics. John Wiley \& Sons, NY.

ReliaSoft Corporation (2006). http://www.weibull.com/LifeDataWeb/ characteristic_effects_of_the_shape_parameter,_beta,_for_the_ weibull_distribution.htm.

Rentch JS, Desta F, Miller GW (2002). Climate, canopy disturbance, and radial growth averaging in a second-growth mixed-oak forest in West Virginia, USA. Can. J. For. Res. 32, 915-927.

Stokes MA, Smiley TL (1968). An Introduction to Tree-ring Dating. The University of Arizona, Tucson, Arizona.

Su HC (1980). Studies on the rare and threatened forest plants of 
Taiwan. Bull. Exp. For. Nat. Taiwan Univ. 125, 126-205. (in Chinese with an English abstract).

Tanouchi H, Yamamoto S (1995). Structure and regeneration of canopy species in an old-growth evergreen broad-leaved forest in Aya district, southwestern Japan. Vegetatio 117, 51-60.

Wu DY (1992). Studies on the Population Structure in Amentotaxus formosana Li. Master Thesis. Graduate Institute of Forestry, National Taiwan University. pp. 56-60. (in Chinese with an English abstract).
Yang SZ (1994). Studies on the vegetation ecology of Chachayalaishan Nature Reserve for Amentotaxus formosan in southern Taiwan. Quart. J. Chin. For. 27, 3-18. (in Chinese with an English abstract).

Yang SZ (1996). Study of Vegetation Ecology of Amentotaxus formosana Li (Taxaceae). Doctoral Dissertation. Graduate Institute of Forestry, National Taiwan University. pp. 62-73. (in Chinese with an English abstract).

(Handling editor: Jin-Zhong Cui) 